\title{
Planejamento Estático de Sistemas de Transmissão de Energia Elétrica Utilizando Um Novo Índice de Sensibilidade
}

\author{
Augusto C. L. Oliveira*. Isabela M. Mendonça** \\ Felipe G. Duque**
*Curso de Engenharia Mecatrônica, Instituto Federal de Educação, Ciência e Tecnologia do Sudeste de Minas Gerais Rua Bernardo Mascarenhas, 1283, Bairro Fábrica, 36080-001, Juiz de Fora, MG, Brasil
(Tel: 05532 4009-3009; e-mail: alaviola11@gmail.com)
**Núcleo de Eletricidade, Instituto Federal de Educação, Ciência e Tecnologia do Sudeste de Minas Gerais Rua Bernardo Mascarenhas, 1283, Bairro Fábrica, 36080-001, Juiz de Fora, MG, Brasil
(e-mails: isabela.mendonca@ifsudestemg.edu.br,felipe.duque@ifsudestemg.edu.br)

\begin{abstract}
This paper presents a new technique for solving the problem of planning the expansion of electric power transmission systems. It is suggested here a methodology associated on the analysis and proposition of an expansion parameter, based the hyperbolic tangent function, and of a sensitivity indicator, elaborated from the statistic selection and combination of indexes there is in technical literature, so that, applied to a constructive heuristic algorithm in which the expansion decisions are relaxed, good results are gotten. The solutions found were satisfactory for the planning horizon adopted didactic systems of small and medium size, and, therefore, it is a robust and efficient tool.
\end{abstract}

Resumo: Este artigo apresenta uma nova técnica de resolução do problema de planejamento da expansão de sistemas de transmissão de energia elétrica. Sugere-se aqui uma metodologia relacionada a análise e proposição de um parâmetro de expansão, baseado na função tangente hiperbólica, e de um indicativo de sensibilidade, elaborado a partir da seleção estatística e combinação de índices existentes na literatura técnica, de modo que, aplicados a um algoritmo heurístico construtivo no qual as decisões de expansão são relaxadas, obtenham-se bons resultados. As soluções encontradas foram satisfatórias para o horizonte de planejamento adotados sistemas didáticos de pequeno e médio porte, e constitui, portanto, uma ferramenta robusta e eficiente.

Keywords: planning; transmission; optimization; sensibility; expansion; heuristic.

Palavras-chaves: planejamento; transmissão; otimização; sensibilidade; expansão; heurística.

\section{INTRODUÇÃO}

O Sistema Elétrico Brasileiro destaca-se pela geração de energia elétrica, basicamente, por meio de usinas hidroelétricas, conforme dados explícitos na Fig. 1, coletados no Banco de Informações de Geração (BIG), da Agência Nacional de Energia Elétrica (ANEEL) (ANEEL, 2018).

Essa característica deve-se ao grande potencial das bacias hidráulicas existentes ao longo de todo o país, haja vista a diversidade de ciclos hidrológicos, diferentemente do que ocorre no âmbito mundial, onde as principais fontes de geração não são renováveis, mas provenientes da queima de combustíveis fósseis como carvão, óleo e gás natural em termelétricas (EPE, 2018).

Além disso, como o Brasil apresenta elevadas dimensões territoriais, mais precisamente $8.515 .759,090 \mathrm{~km}^{2}$ de área superficial, segundo o Instituto Brasileiro de Geografia e Estatística (IBGE), há vastas distâncias entre as unidades geradoras e os centros consumidores, o que torna o problema de Planejamento da Expansão da Transmissão (PET) uma tarefa complexa em virtude do transporte de blocos de energia a fim de garantir o suprimento dos consumidores a um menor custo.

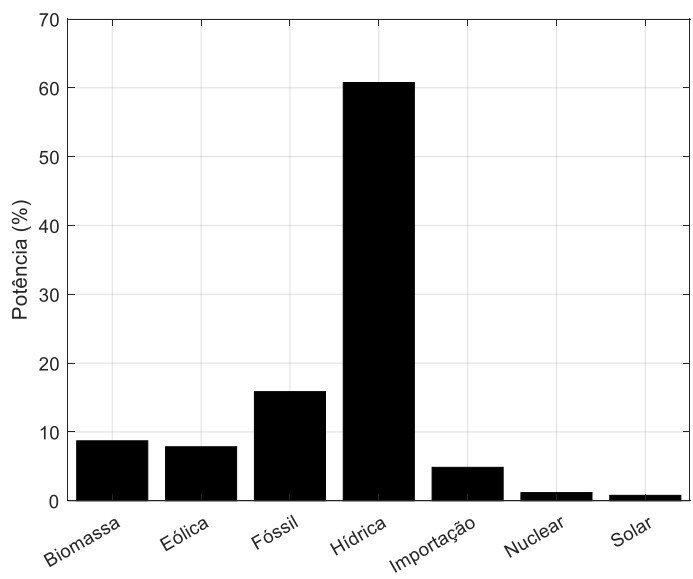

Fig. 1 Geração de Energia Elétrica por Fonte no Brasil. 
Dessa forma, as dificuldades em encontrar soluções para o PET existem por se tratar de um problema de programação não linear inteira mista com algumas particularidades, sendo elas: (i) a possibilidade de existência de sistemas não conexos, ou seja, que possuem barras ilhadas; (ii) região de solução não convexa; (iii) explosão combinatória referente às alternativas de investimento, que causam elevado esforço computacional para a determinação, dentre um conjunto prédefinido de circuitos candidatos, aqueles que construídos otimizam o investimento.

Portanto, mediante a esse contexto, elaborar algoritmos capazes de encontrar a solução do problema de maneira eficiente não é algo fácil.

$\mathrm{Na}$ literatura especializada, Romero et al. (2003) analisou o problema do PET por meio da aplicação de um algoritmo heurístico construtivo (AHC) no modelo de transporte inicialmente proposto por Garver. E, dando continuidade a esse trabalho, Romero et al. (2005) realizou testes a fim de mostrar a eficiência do modelo CC para sistemas conhecidos.

Também foram propostos algoritmos que envolvem modelagens de fluxo de potência ideal (FPI) e utilização da técnica do ponto interior primal-dual (Oliveira, et al., 2005), que apresentam parâmetros de decisão fuzzy para solucionar problemas críticos encontrados no PET (Sousa e Asada, 2009), que utilizam ferramentas híbridas associadas AHC e a otimização por meio de enxame de partículas (PSO) (Gomes e Saraiva, 2015), dentre outros.

Em meio a todo contexto abordado, o objetivo deste trabalho se enquadra no desenvolvimento de uma heurística de análise e proposição de um parâmetro de expansão $(P E)$ e de um índice de sensibilidade que aplicados a um AHC obtenham-se bons conjuntos soluções a baixos esforços computacionais.

\section{FORMULAÇÃO E METODOLOGIA}

O AHC utilizado para o desenvolvimento deste artigo foi proposto por Mendonça (2016) e tem por finalidade proporcionar a obtenção de soluções de investimento economicamente viáveis para o problema do PET, bem como os planos finais de expansão de cada um dos sistemas analisados. Sendo assim, é importante salientar que:

(i) $\mathrm{O}$ algoritmo heurístico utiliza como técnica de solução a metodologia primal-dual dos pontos interiores (Granville, 1994), permitindo a inclusão de não linearidades intrínsecas ao PET.

(ii) A decisão da expansão é realizada por meio da variação de um parâmetro de expansão $(P E)$ e a inclinação da função tangente hiperbólica é inserida ao problema de otimização por meio do modelo CC de fluxo de carga (Silva Junior, 2003).

(iii) É analisada a sensibilidade a partir do cálculo de um índice para cada caminho candidato e aquele que possuir maior valor é selecionado por ser o mais atrativo.

(iv) Cada vez que uma linha é selecionada mediante análise do item (iii) a operação do sistema é verificada e se o corte de carga for maior que uma tolerância pré-estipulada (1 MW) o processo se repete até que ele fique abaixo dela.

(v) Em sistemas não conexos, ou seja, naqueles que possuem barras isoladas, são inseridas linhas fictícias com baixas susceptâncias quando comparadas as das linhas candidatas (relação entre elas é de $10^{-3}$ ).

(vi) Em cada barra de carga possui um gerador fictício que atende a demanda a altos custos quando ela não é garantida pelas expansões realizadas.

Portanto, o modelo matemático que descreve as características supracitadas é representado pelas seguintes equações (1 a 11), nas quais é dado por $\mathrm{E}$ o conjunto das linhas existentes na topologia base de um sistema, $\mathrm{C}$ o conjunto de linhas candidatas à expansão e por $\mathrm{F}$ o conjunto das linhas fictícias:

$$
\begin{gathered}
\text { Min } \sum_{i=1}^{n r} c_{i} \cdot r_{i}+\sum_{l \in \Omega C} c_{l} \cdot P E_{l} \\
g_{i}+r_{i}-\sum_{j \in \Omega C} f_{l}=d_{i} \\
0 \leq g_{i} \leq \bar{g}_{i} \\
0 \leq r_{i} \leq \bar{r}_{i} \\
0 \leq P E_{l} \leq 1, \forall(l) \in \Omega C \\
f_{l}=-\gamma_{l} \theta_{l}, \forall(l) \in \Omega E \\
f_{l}=-\gamma_{l_{F I C}} \varphi_{l}, \forall(l) \in \Omega F \\
\gamma_{l_{F I C}}<\gamma_{l}, \forall(l) \in \Omega F \\
\varphi_{l}=-P E_{l} \gamma_{l} \theta_{l}, \forall(l) \in \Omega C \\
\bar{f}_{l} \\
\left|f_{l}\right| \leq \bar{f}_{l}, \forall(l) \in \Omega E \Omega C \\
\end{gathered}
$$

Onde:

$n r$ - Número de geradores fictícios.

$c_{i}$ - Custo do déficit de energia (US\$/MW) por ano.

$c_{l}$ - Custo de construção da linha candidata $l$ (US\$) por ano.

$g_{i}$ - Geração da unidade geradora na barra $i(\mathrm{MW})$.

$\bar{g}_{i}$ - Limite máximo de geração na barra $i$ (MW).

$r_{i}$ - Geração da unidade geradora fictícia na barra $i$ (MW)

$\bar{r}_{i}$ - Limite máximo da geração fictícia na barra $i(\mathrm{MW})$. 
$\Omega i$ - Conjunto de barras conectadas às barras $i$.

$P E_{l}$ - Parâmetro de expansão da linha candidata $l$, variável inteira $0 / 1$.

$f_{l}$ - Fluxo de potência ativa da linha $l(\mathrm{MW})$.

$\bar{f}_{l}$ - Limite de fluxo de potência ativa da linha $l$ (MW).

$\gamma_{l}$ - Susceptância da linha $l$.

$d_{i}$ - Demanda na barra $i$ (MW).

$\varphi_{l}$ - Diferença angular entre as barras da linha fictícia $l$.

$\theta_{l}$ - Diferença angular entre as barras da linha $l$.

$\gamma_{l_{F I C}}$ - Susceptância da linha fictícia $l$.

\subsection{Parâmetro de Expansão Proposto}

A decisão de construir ou não determinadas linhas a fim de sanar o problema do PET é representado pelo $P E$, em que, se $P E=0$, opta-se pela não construção, se $P E=1$, opta-se pela construção. No entanto, tal parâmetro trata-se de uma variável discreta, o que poderia causar dificuldades na resolução de problemas de programação inteira.

Logo, foram adotados por Silva Junior (2003) e Oliveira et al. (2005) uma heurística no qual o $P E$ possa assumir valores contínuos no intervalo $[0,1]$, a partir de uma função tangente hiperbólica (Tanh), ou seja, uma função que apresenta comportamento parecido com a função degrau (modelagem ideal para o $P E$ ).

Baseado nisso, Mendonça (2016) propôs uma heurística, na qual é utilizada a função tangente hiperbólica modificada, (12), com o "x" variando de 0 a 20 e "A" variando de 0,1 a 6 com um incremento de 0,1 .

$$
P E=\frac{e^{x / A}-1}{e^{x / A}+1}, \forall 0 \leq x \leq 20
$$

Na medida em que "A" cresce, a inclinação da curva é alterada, Fig. 2; o que torna possível a execução do algoritmo para diferentes sensibilidades da função $P E$, com o objetivo de direcionar a busca para regiões que possuem soluções de boa qualidade.

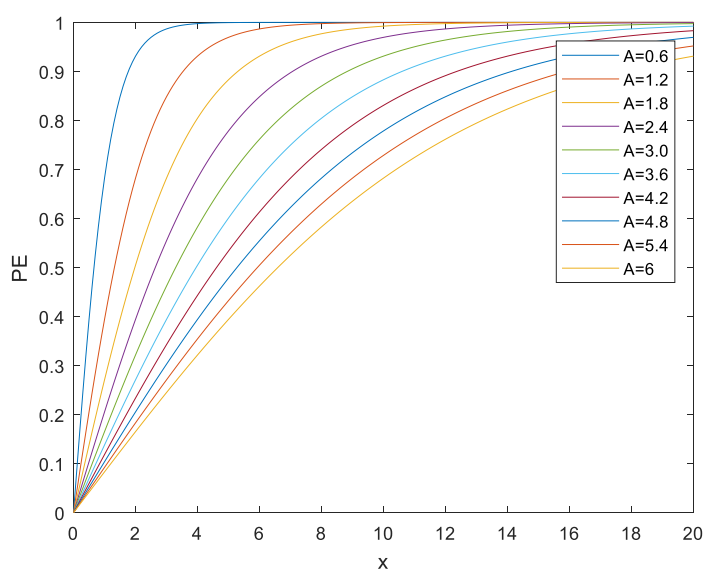

Fig. 2 Exemplo Ilustrativo das Variações da Função Tanh.
Portanto, com o intuito de realizar uma varredura refinada próxima ao degrau unitário, mapear novos pontos dessa região e promover a redução do esforço computacional sem perder a boa sensibilidade da Tanh é proposta uma nova modelagem de $P E$ fundamentada na razão de dois polinômios de mesma ordem. Para isso, foram gerados vetores domínio e imagem da Tanh no intervalo de interesse (Fig. 2) e empregado o método numérico Non-linear Least Squares aliado ao algoritmo Trust-region para determinação da função (13) que melhor os descrevem.

$$
\begin{gathered}
P E_{p}=\frac{\left(\frac{x}{A}\right)^{5}+p_{1}\left(\frac{x}{A}\right)^{4}+p_{2}\left(\frac{x}{A}\right)^{3}+p_{3}\left(\frac{x}{A}\right)^{2}+p_{4}\left(\frac{x}{A}\right)+p_{5}}{\left(\frac{x}{A}\right)^{5}+q_{1}\left(\frac{x}{A}\right)^{4}+q_{2}\left(\frac{x}{A}\right)^{3}+q_{3}\left(\frac{x}{A}\right)^{2}+q_{4}\left(\frac{x}{A}\right)+q_{5}} \\
p_{1}=0,2444 ; p_{2}=5,963 ; p_{3}=8,448 ; p_{4}=17,13 ; p_{5}=-0,002475 \\
q_{1}=0,2456 ; q_{2}=5,8777 ; q_{3}=9,782 ; q_{4}=9,111 ; q_{5}=17,03
\end{gathered}
$$

Através da variação de sensibilidade do $P E$ proposto obtevese um emaranhado de curvas (Fig. 3):

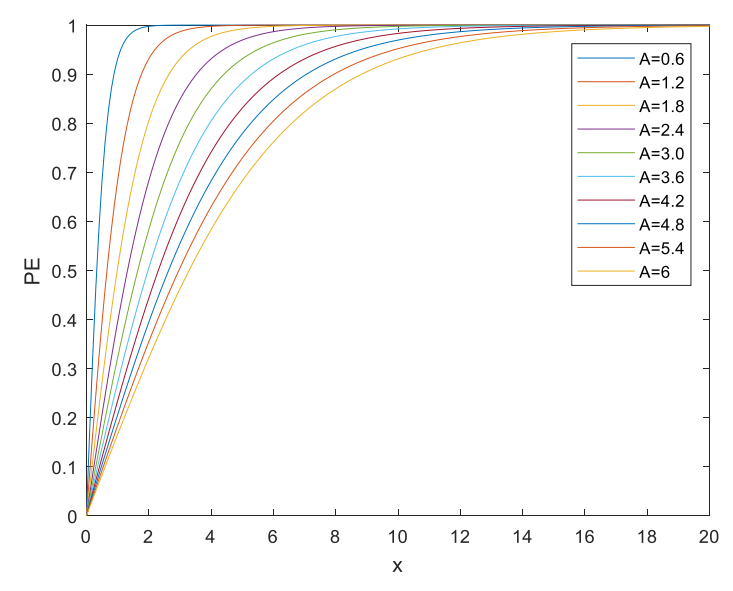

Fig. 3 Exemplo Ilustrativo da Função $P E$ proposta.

Dessa forma, o AHC utilizado é dividido em etapa contínua obtenção dos valores contínuos do PE de todas as linhas candidatas, para cada valor de "A" - e etapa discreta consiste em definir quais linhas candidatas serão construídas, usando a metodologia dos índices de sensibilidade.

\section{2 Índice de Sensibilidade Proposto}

Neste artigo serão analisados seis índices de sensibilidade usuais em trabalhos científicos para sistemas de transmissão de pequeno, médio e grande porte. A partir dos resultados encontrados para cada um deles, mediante a simulação do AHC previamente apresentado, será proposto um novo índice que consistirá na combinação daqueles com melhores desempenhos, adotados alguns critérios de seleção.

As identificações desses seis índices, bem como suas respectivas formulações, seguem em (14), (15), (16), (17), (18) e (19):

\section{Sensibilidade Baseada em Garver (GA1)}

$$
I S_{l}^{g a 1}=-P E_{l} \gamma_{l} \theta_{l}, \forall(l) \in C
$$


GA1 Modificada pelo Custo (GA2)

$$
I S_{l}^{g a 2}=\frac{-P E_{l} \gamma_{l} \theta_{l}}{c_{l}}, \forall(l) \in C
$$

Sensibilidade Baseada no Mínimo Esforço (ME1)

$$
I S_{l}^{m e 1}=P E_{l} \frac{1}{2} \gamma_{l} \theta_{l}^{2}, \forall(l) \in C
$$

ME1 Modificada pelo Custo (ME2)

$$
I S_{l}^{m e 2}=\frac{P E_{l} \frac{1}{2} \gamma_{l} \theta_{l}^{2}}{c_{l}}, \forall(l) \in C
$$

Sensibilidade Baseada no Mínimo Corte de Carga (MCC1)

$$
I S_{l}^{m c c 1}=P E_{l}\left(\theta_{l}\right)\left(\lambda_{l}\right), \forall(l) \in C
$$

MCC1 Modificado pelo Custo (MCC2)

$$
I S_{l}^{m c c 2}=\frac{P E_{l}\left(\theta_{l}\right)\left(\lambda_{l}\right)}{c_{l}}, \forall(l) \in C
$$

Em que:

\section{$\lambda_{l}$ é o multiplicador de Lagrange.}

As sensibilidades baseadas em Garver, (14) e (15), buscam priorizar linhas que possuem o maior valor absoluto de fluxo de potência ativa. Já aquelas relacionadas ao mínimo esforço, (16) e (17), visam a minimização dos cortes de carga no sistema elétrico a partir da representação de unidades geradoras artificiais no problema de otimização tornando sua solução sempre viável. E, por fim, as pertinentes ao mínimo corte de carga, (18) e (19), permitem a localização de linhas que produzem maior diminuição do corte de carga do sistema.

De acordo com o fluxograma da Fig. 4, para a aplicação de cada um dos índices apresentados no AHC são obtidos conjuntos de possíveis soluções para cada um dos sistemas de transmissão simulados.

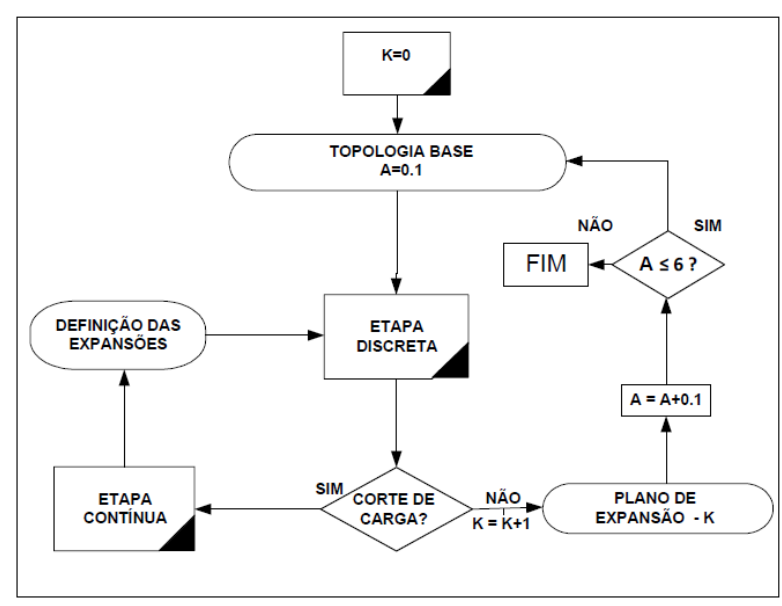

Fig. 4 Fluxograma do AHC.

De posse desses dados, algumas características podem ser utilizadas a fim de qualificar os melhores índices a nível de cada sistema, sendo elas vinculadas algebricamente às seguintes perguntas, (a), (b) e (c):

(a) A otimalidade foi atingida? É importante a verificação dessa característica, uma vez que o principal objetivo PET é solucionar o problema de cortes de carga a um menor custo. Dessa forma, torna-se interessante a obtenção da otimalidade para os planos finais de expansão a fim de melhor validar a técnica sugerida e compará-la com dados publicados na literatura. Segue a formulação matemática que determina a proximidade do menor valor de investimento do conjunto à otimalidade, $R_{1},(20)$ :

$$
R_{1}=100 \frac{V_{\min }-V_{o}}{V_{o}}
$$

(b) O quão distante a média das amostras se encontra da otimalidade? Como um índice de sensibilidade apresenta caráter local, ou seja, possui dificuldades em obter ótimas soluções globais, a análise dessa característica torna-se relevante, visto que muitas vezes apenas um subconjunto pequeno de amostras possui baixos valores de investimento quando comparados a otimalidade. E, como será proposta a criação de um índice a partir da combinação de demais índices, é viável a seleção daqueles que apresentam esse subconjunto o mais próximo do espaço amostral, isto é, com uma média (21) que tangencie à otimalidade. Logo, a representação matemática dessa característica é $R_{2},(22)$ :

$$
\begin{gathered}
\bar{V}=\frac{\sum_{n=1}^{N} V_{n}}{N} \\
R_{2}=100 \frac{\bar{V}-V_{o}}{V_{o}}
\end{gathered}
$$

(c) Qual a dispersão do conjunto de resultados? A fim de verificar a convergência do índice adotado e tornar consistente a característica anterior (b), é necessário analisar a dispersão dos resultados em relação à média das amostras. Os conceitos estatísticos utilizados são desvio padrão (23) e, a partir dele, coeficiente de variação, conforme explícito por $R_{3},(24)$ :

$$
\begin{gathered}
\sigma=\sqrt{\frac{\sum_{n=1}^{N}\left(V_{n}-\bar{V}\right)^{2}}{N-1}} \\
R_{3}=100 \frac{\sigma}{\bar{V}}
\end{gathered}
$$

Onde:

$N$ - Número total de amostras.

$V_{\text {min }}$ - Valor mínimo do conjunto de amostras.

$V_{n}-$ Valor de investimento da amostra.

$\bar{V}$ - Valor médio do conjunto amostral.

$V_{o}$ - Otimalidade difundida na literatura técnica.

$\sigma$ - Desvio padrão das amostras. 
$R_{1}$ - Qualificação percentual da característica (a).

$R_{2}$ - Qualificação percentual da característica (b).

$R_{3}$ - Qualificação percentual da característica (c).

Vale ressaltar que, para sistemas em que a otimalidade é desconhecida, o valor de $V_{o}$ pode ser considerado como a solução contínua do problema de planejamento, visto que, apesar de inviável, é a melhor possível.

Definidas as três características, é possível qualificar cada índice de sensibilidade " $i$ " a nível de cada sistema de transmissão “ $j ”$ através de (25), $Q_{i j}$ :

$$
Q_{i j}=\sum_{w=1}^{3} p_{w}\left(\frac{R_{w}}{100}\right)_{i j}
$$

Em que:

$Q$ - Matriz de Qualificação.

$p_{w}$ - Grau de importância (peso) da característica $w$.

$R_{w}$ - Qualificação percentual da característica $w$.

Neste trabalho serão adotados $p_{1}=2, p_{2}=4$ e $p_{3}=1$. Tal escolha busca priorizar índices nos quais a média das amostras se encontra próxima da otimalidade, visto que isso é de extrema relevância quando a proposta é a combinação de índices. Além disso, a característica (a) foi melhor ponderada em relação a (c), pois está relacionada diretamente com o objetivo final do trabalho, enquanto a outra trata-se de uma complementação da característica (b), de peso maior.

Logo, por meio da apresentação gráfica dos resultados de cada uma das colunas de $Q$, é possível encontrar os melhores índices para cada um dos sistemas simulados através da seleção daqueles com valores de qualificação mais próximos de zero, e, assim, definir um novo índice compostos por eles.

\section{ESTUDO DE CASOS}

Nesta seção será desenvolvido um estudo de casos para aplicação da metodologia proposta em 3 sistemas de transmissão comumente utilizados na literatura para validação de novas técnicas. As soluções ótimas globais conhecidas para cada um deles encontram-se na Tabela 1:

Tabela 1. Valores ótimos globais difundidos na literatura.

\begin{tabular}{c|c}
\hline Sistema de Transmissão & Valores ótimos (US\$) \\
\hline Garver com redespacho & $110.000 .000,00$ \\
\hline Garver sem redespacho & $200.000 .000,00$ \\
\hline IEEE 24 barras cenário G1 & $390.000 .000,00$ \\
\hline
\end{tabular}

O sistema Garver não conexo é formado por 6 barras, 6 circuitos existentes na topologia base, 15 circuitos candidatos à expansão e demanda prevista de 760 MW (Garver, 1970).

Há dois casos para o sistema Garver: (i) com redespacho de geração, onde considera-se um número máximo de 3 expansões por caminho candidato e, consequentemente, um total de $4^{15}$ combinações de possíveis alternativas de investimentos; (ii) sem redespacho de geração, em que a dificuldade do PET aumenta, visto que as unidades geradoras têm seus despachos de potência pré-determinados, e considera-se um limite de 4 expansões por caminho candidato ( $5^{15}$ possíveis combinações de investimento).

Por sua vez, o sistema IEEE 24 barras cenário G1 possui 38 circuitos existentes na topologia base, 41 candidatos, demanda prevista de 8550 MW e geração de 10215 MW sem reprogramação (Romero et al., 2005). Para esse sistema existem $4^{41}$ combinações de investimento (máximo 3 expansões por caminho candidato).

Logo, realizadas as simulações, foi obtida a qualificação dos indicativos de sensibilidade para cada um desses sistemas mediante aplicação das análises propostas (25). Os resultados alcançados, que são adimensionais e normalizados em relação ao valor máximo, estão explícitos na Fig. 5.

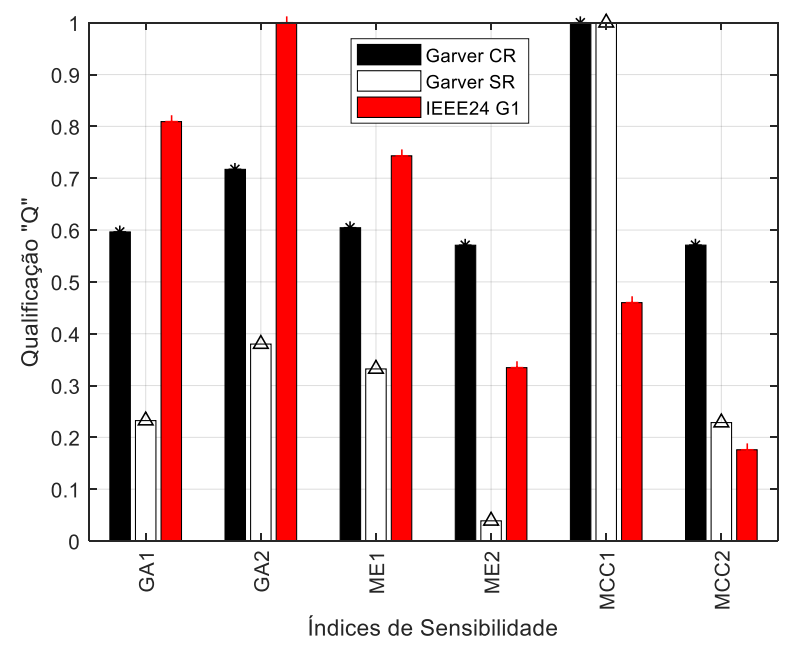

Fig. 5 Qualificação dos índices de sensibilidade.

Com base na disposição dos dados, verifica-se que os melhores índices, isto é, aqueles com valores de qualificação ( $Q)$ mais próximos de zero, para cada sistema analisado são: (i) Garver com redespacho: $I S^{m c c 2}, I S^{m e 2}, I S^{g a l}$ e $I S^{m e l}$; (ii) Garver sem redespacho: $I S^{m e 2}, I S^{m c c 2}$ e $I S^{\text {gal }}$; (iii) IEEE 24 barras cenário G1: $I S^{m c c 2}$ e $I S^{m e 2}$.

Dessa forma, como os índices $I S^{m e 2}, I S^{\text {mcc2 }}$ e $I S^{\text {gal }}$ foram eficientes na maioria dos sistemas adotados, foi criado um indicativo de sensibilidade, $I S^{P I}$, por meio da combinação deles. Segue em (26) a sua formulação:

$$
\begin{gathered}
I S_{l}{ }^{P 1}=\left(\frac{\sum_{i=1}^{3} x_{\max } \frac{\left|I S_{l}^{i}\right|}{I S_{\max }^{i}}}{3}\right), \forall(l) \in C \\
i_{1}=I S_{l}^{m e 2}, i_{2}=I S_{l}^{m c c 2}, i_{3}=I S_{l}^{g a 1}
\end{gathered}
$$

\section{ANÁLISE DE DESEMPENHO DO NOVO ÍNDICE}

O índice proposto atingiu a otimalidade (Tabela 1) para os sistemas Garver e apresentou um valor de investimento de US\$ 420.000.000,00 para o IEEE 24 barras cenário G1. 
Comparando, em relação às características (a), (b) e (c), os resultados encontrados para a metodologia proposta com aqueles obtidos por Mendonça (2016), em que foi adotado o índice portfólio, $I S^{P}$, combinação entre os seis índices aqui estudados, têm-se (Tabela 2):

Tabela 2. Comparativo entre índices: características (a), (b) e (c).

\begin{tabular}{c|c|c}
\hline Sistema de Transmissão & $I \boldsymbol{S}^{\boldsymbol{P}}$ & $\boldsymbol{I S}^{\boldsymbol{P I}}$ \\
\hline \multicolumn{3}{c}{ Característica (a) } \\
\hline Garver com redespacho & Sim & Sim \\
\hline Garver sem redespacho & Sim & Sim \\
\hline IEEE 24 barras cenário G1 & $4,1 \%$ & $7,7 \%$ \\
\hline \multicolumn{3}{c}{ Característica (b) } \\
\hline Garver com redespacho & $19,9 \%$ & $20,3 \%$ \\
\hline Garver sem redespacho & $12,7 \%$ & $4 \%$ \\
\hline IEEE 24 barras cenário G1 & $9,4 \%$ & $12 \%$ \\
\hline \multicolumn{3}{c}{ Característica (c) } \\
\hline Garver com redespacho & $8,7 \%$ & $6,9 \%$ \\
\hline Garver sem redespacho & $12 \%$ & $7 \%$ \\
\hline IEEE 24 barras cenário G1 & $2,2 \%$ & $0,5 \%$ \\
\hline
\end{tabular}

Verifica-se na Tabela 2 que as duas metodologias atingiram a otimalidade para os sistemas Garver, porém o $I S^{P}$ foi ligeiramente melhor nesse quesito para o IEEE 24 barras cenário G1.

Quanto à média, os resultados entre os índices foram bem equilibrados, com destaque para o $I S^{P I}$ no sistema Garver sem redespacho. Ressalta-se que a obtenção de bons resultados com base nessa característica pode ser interessante para demonstrar a qualidade de um conjunto de rotas relevantes em caso de implementações futuras de otimização bioinspirada.

Por fim, a aplicação das heurísticas e análises propostas garantiram para todos os sistemas uma convergência melhor quando comparada àquela em que se faz o uso do índice portfólio, visto que o valor máximo do coeficiente de variação foi de $7 \%$.

\section{CONCLUSÕES}

Conforme apresentado, a metodologia proposta juntamente com o algoritmo heurístico construtivo adotado ofereceu soluções eficientes quando comparadas àquelas presentes na literatura.

Além disso, ao comparar o novo índice $\left(I S^{P l}\right)$ ao portfólio sugerido por Mendonça (2016), é possível verificar o seu bom desempenho no que diz respeito às características (a), (b) e (c) analisadas, haja vista a combinação de apenas três indicativos de sensibilidade e a implementação de uma nova função parâmetro de expansão na etapa contínua do AHC.

Contudo, mesmo que bons resultados tenham sido obtidos pela nova estratégia de planejamento da expansão, como em toda metodologia heurística ou metaheurística, não é possível garantir a otimalidade, mas sim a viabilidade das soluções encontradas. Dessa forma, são sugeridos trabalhos futuros de implementação das técnicas propostas para demais sistemas, por exemplo, o sul brasileiro e colombiano, bem como sua utilização com primeiro estágio de resolução do problema do PET por meio de otimização bioinspirada.

\section{AGRADECIMENTOS}

Os autores agradecem aos órgãos de fomento à pesquisa FAPEMIG e CNPq e ao IF Sudeste MG - Campus Juiz de Fora, em especial à Diretoria de Pesquisa, Inovação e PósGraduação (DPIPG), pelo apoio fundamental para o desenvolvimento deste trabalho.

\section{REFERÊNCIAS}

ANEEL, Agência Nacional de Energia Elétrica. (2018) "Banco de Informações de Geração," Disponível em: <http://www2.aneel.gov.br/aplicacoes/capacidadebra sil/capacidadebrasil.cf m>. Acesso em: 23 ago. 2018.

EPE, Empresa de Pesquisa Energética. (2018) "Matriz energética e elétrica," Disponível em: <http://www.epe.g ov.br/pt/abcdenergia >. Acesso em: 23 ago. 2018.

Garver, L. L. (1970) "Transmission Network Estimation Using Linear Programming," IEEE Transactions On Power Apparatus And Systems, v. 89, n. 7, p.1688-1697.

Gomes, P. V. and Saraiva, J. T. (2015) "Static transmission expansion planning using Heuristic and metaheuristic techniques," in PowerTech, 2015 IEEE Eindhoven, pp. $1-6$

Granville, S. (1994) "Optimal Reactive Dispatch Through Interior Point Methods," IEEE Transactions on Power Systems, vol. 9, No. 1, pp. 136-146.

Mendonça, I. M. (2016) "Identificação de Rotas Relevantes Para o Planejamento Estático da Expansão de Sistemas de Transmissão de Energia Elétrica," Tese de Doutorado, UFJF.

Oliveira, E. J., da Silva I. C., Pereira J. L., and Carneiro, S (2005) "Transmission System Expansion Planning Using a Sigmoid Function to Handle Integer Investment Variables," IEEE Transactions on Power Systems, vol. 20, no. 3, pp. 1616-1621, Aug. 2005.

Romero, R. Rocha, C., Mantovani, M., and Mantovani, J. R. S. (2003) "Analysis of Heuristic Algorithms for the Transportation Model in Static and Multistage Planning in Network Expansion Systems," Generation, Transmission and Distribution, IEE Proceedings, vol. 150, No. 5, pp 521-526.

Romero, R., Rocha, C. Mantovani, J. R. S., and Sanchez, I. G. (2005) "Constructive Heuristic Algorithm for the DC Model in Network Transmission Expansion Planning," Generation, Transmission and Distribution, IEE Proceedings, vol. 152, No. 2, pp. 277-282.

Silva Junior, I. C. (2003) "Planejamento Estático Da Expansão De Sistemas de Transmissão Utilizando Um Novo Algoritmo Heurístico Construtivo," Dissertação, UFJF.

Sousa, A. S. and Asada, E. N. (2009) "Fuzzy Guided Constructive Heuristic Applied to Transmission System Expansion Planning," International Conference on Intelligent System Applications to Power Systems, pp.16. 